José de Jesús Leal Carvajalino

\title{
Acoplamento de Estados Limites na Avaliação da Confiabilidade Estrutural de Dutos e Estruturas
}

Tese de Doutorado

Tese apresentada como requisito parcial para obtenção do grau de Doutor pelo Programa de Pós-Graduação em Engenharia Mecânica do Departamento de Engenharia Mecânica do Centro Técnico Científico da PUC-Rio.

Orientador: José Luiz de França Freire 


\section{Acoplamento de Estados Limites na Avaliação da Confiabilidade Estrutural de Dutos e Estruturas}

Tese de Doutorado

Tese apresentada como requisito parcial para obtenção do grau de Doutor pelo Programa de Pós-Graduação em Engenharia Mecânica do Centro Técnico Científico da PUC-Rio. Aprovada pela Comissão Examinadora abaixo assinada.

Prof. José Luiz de França Freire

Orientador

Departamento de Engenharia Mecânica - PUC-Rio

Prof. Marco Antônio Meggiolaro

Departamento de Engenharia Mecânica - PUC-Rio

Prof. Arthur Martins Barbosa Braga Departamento de Engenharia Mecânica - PUC-Rio

Prof. Jaime Tupiassú Pinho de Castro Departamento de Engenharia Mecânica - PUC-Rio

Prof. Tito Luiz da Silveira

Universidade Federal do Rio de Janeiro

Prof. Jose Antonio da Cunha Ponciano Gomes

Universidade Federal do Rio de Janeiro

Prof. José Eugenio Leal

Coordenador Setorial do Centro Técnico Científico - PUC-Rio

Rio de Janeiro, 29 de Abril de 2010 
Todos os direitos reservados. É proibida a reprodução total ou parcial do trabalho sem a autorização da universidade, do autor e do orientador.

\section{José de Jesús Leal Carvajalino}

Graduou-se em Engenharia Metalúrgica na Universidad Industrial de Santander (Bucaramanga, Colômbia) em 2000. Mestre em Engenharia Mecânica pela Pontifícia Universidade Católica do Rio de Janeiro em 2004.

Ficha Catalográfica

Carvajalino, José de Jesús Leal

Acoplamento de estados limites na avaliação da confiabilidade estrutural de dutos e estruturas / José de Jesús Leal Carvajalino ; orientador: José Luiz de França Freire. -2010.

139 f. : il. (color.) ; $30 \mathrm{~cm}$

Tese (doutorado)-Pontifícia Universidade Católica do Rio de Janeiro, Departamento de Engenharia Mecânica, 2010.

Inclui bibliografia

1. Engenharia mecânica - Teses. 2. Confiabilidade estrutural. 3. Distribuição multinormal. 4. Dutos. 5. Corrosão. 6. Mossa. 7. Sulco. I. Freire, José Luiz de França. II. Pontifícia Universidade Católica do Rio de Janeiro. Departamento de Engenharia Mecânica. III. Título. 
Para mis papás, Jesús y Antonia, mis hermanos, Helio y Francisca, y mis amores, Saori y Kei Antônio. 


\section{Agradecimentos}

Ao professor José Luiz de França Freire, pela orientação e por todos os ensinamentos durante todos estes anos, muito obrigado.

Ao CNPq e à CAPES pelos auxílios concedidos.

Aos meus amigos e colegas Marco, Leonardo, José Luiz e Sabrina da PRIMA-7S Integridade Estrutural.

Ao professor Ivan Uribe da UIS-Colombia.

Aos professores da PUC-Rio, da engenharia mecânica e em particular aos professores Arthur Braga, Jaime Castro e Ronaldo Vieira.

Aos professores membros da banca examinadora.

A todos os colegas da pós-graduação, aos do Laboratório de Fotomecânica da PUC-Rio, Leo, Marcos, Gerardo, Jorge, Maira, Gilmar e tantos outros que passaram por aqui.

A todos os funcionários do departamento de engenharia mecânica, pela ajuda brindada durante este tempo, especialmente ao Garcia.

À Pontifícia Universidade Católica do Rio de Janeiro, e seus funcionários em geral. 


\section{Resumo}

Carvajalino, José de Jesús Leal; Freire, José Luiz de França. Acoplamento de Estados Limites na Avaliação da Confiabilidade Estrutural de Dutos e Estruturas. Rio de Janeiro, 2010. 139 p. Tese de Doutorado Departamento de Engenharia Mecânica, Pontifícia Universidade Católica do Rio de Janeiro.

Neste trabalho são apresentados conceitos usados na avaliação da confiabilidade estrutural com o objetivo de calcular a probabilidade de falha de uma estrutura enquanto ela atende aos fins para os quais foi projetada durante sua vida útil. Uma metodologia de análise de confiabilidade estrutural foi desenvolvida, tendo como foco os dutos de transporte de óleo e gás natural, embora possa ser aplicada a diferentes equipamentos. A metodologia permite o acoplamento de diferentes eventos que possam ocorrer na vida de uma estrutura. Entende-se por eventos a aparição de defeitos por diferentes vias: processos corrosivos, danos por terceiros, operações incorretas, etc., ou, eventos relacionados à inspeção da estrutura, duto ou equipamento. Cada evento é descrito por uma função de estado limite do tipo capacidade x demanda. $\mathrm{O}$ acoplamento desses estados limites é dado pela união ou interseção deles (sistemas em série, paralelo, ou combinação deles). A análise é reduzida ao cálculo da confiabilidade de um sistema, cuja solução é feita usando a função padrão multinormal e os métodos de primeira ordem FORM, para o cálculo da probabilidade de falha de cada estado limite, e os métodos do produto das probabilidades condicionais $P C M$ e I-PCM, para o cálculo da probabilidade de falha do sistema através da integral multinormal. As informações obtidas dos resultados desta metodologia podem ser úteis na geração de planos de inspeção, análises preditivas e análises de risco, para contribuir na tomada de decisões sobre prazos e técnicas de inspeção a serem empregadas. A metodologia mencionada acima pode ser implementada em um programa de gerenciamento de confiabilidade estrutural, o qual deve ser capaz de acoplar todos os eventos, os dados conhecidos, as incertezas próprias dos dados e as novas informações ao longo da vida útil de uma estrutura.

\section{Palavras-chave}

Confiabilidade Estrutural; Distribuição Multinormal; Dutos; Corrosão; Mossa; Sulco. 


\section{Abstract}

Carvajalino, José de Jesús Leal; Freire, José Luiz de França (Advisor). Coupling Limit States to Structural Reliability Assessment of Pipelines and Structures. Rio de Janeiro, 2010. 139 p. Doctoral Thesis Departamento de Engenharia Mecânica, Pontifícia Universidade Católica do Rio de Janeiro.

This work presents concepts used in the assessment of structural reliability in order to calculate the probability of failure of a structure as it serves the purposes for which it was designed during their lifetime. A methodology for structural reliability analysis has been developed for the pipeline transportation of oil and natural gas, although, this methodology can be applied to different equipment. The methodology allows the coupling of different events that may occur in the life of a structure. The events can be understood as defects by corrosion, damage by third parties, incorrect operations, etc. or events related to inspection of the structure, pipeline or equipment. Each event is described by a limit state function of the type capacity vs. demand. The coupling of these states limit is given by the union or intersection of these (series systems, parallel systems, or combination of them). The analysis is reduced to system reliability computation and the solution is reached using the integration of the standard multinormal function and first order reliability methods- FORM to calculate the probability of failure of system. The multinormal integral is computation using the product of conditional marginal method- $P C M$ and the improvement of $P C M$ method. The results obtained of this methodology may be useful in the generation of inspection plans and in predictive and risk analysis. The methodology described can be implemented in a structural reliability management program. The program should be able to coupling all events that occur in the lifetime of a pipeline or structure.

\section{Keywords}

Structural Reliability; Multinormal Distribution; Pipelines; Corrosion; Dent; Gouge. 


\section{Sumário}

1. Introdução

15

1.1. Cálculo da probabilidade de falha

1.2. Avaliação da confiabilidade estrutural de dutos com

defeitos de corrosão e danos tipo mossa-sulco

1.3. Conteúdo da tese

2. Conceitos de confiabilidade estrutural

2.1. Probabilidade de falha 28

2.2. Métodos para o cálculo da probabilidade de falha 30

2.2.1. Método de confiabilidade de primeira ordem (form) 30

2.2.2. Método de confiabilidade de segunda ordem (sorm) 34

2.2.3. Novo algoritmo FORM por low e tang (FORM-LT) 34

2.2.4. Método de simulação Monte Carlo 38

2.3. Análise de confiabilidade estrutural de sistemas 39

2.4. Interseção e união de probabilidades $\quad 41$

2.5. Atualização da probabilidade de falha 42

3. Métodos para a solução da função multinormal 44

3.1. A função binormal $\quad 44$

3.2. A função multinormal $\quad 48$

3.3. Método de primeira ordem multinormal-FOMN 51

3.4. Método do produto das probabilidades condicionais - PCM 54

3.5. Método melhorado do produto das probabilidades

condicionais - I-PCM 
4.1. Funções de estados limites $\quad 61$

4.1.1. Medição dos defeitos de corrosão 63

4.1.2. Detecção dos defeitos de corrosão 64

4.2. Falha de um duto corroído 66

4.2.1. Modelos de pressão de falha para dutos corroídos 67

4.2.2. Probabilidade de falha para dutos corroídos 69

4.3. Modelagem da corrosão 76

4.3.1. Modelagem fenomenológica da taxa de corrosão 77

4.3.2. Extrapolação da taxa de corrosão usando só a geometria do defeito $\quad 80$

4.3.3. Aplicação da modelagem da taxa de corrosão na confiabilidade estrutural $\quad 82$

4.4. Atualização segundo o método de inspeção usado 90

5. Dano em dutos causados por interferência externa 95

5.1. Função de estado limite mossa-sulco 95

5.2. Probabilidade de falha do dano mossa-sulco 101

5.2.1. Influência do comprimento do sulco na probabilidade de falha do dano mossa-sulco 106

5.2.2. Influência da pressão de operação na probabilidade de falha do dano mossa-sulco

5.2.3. Influência da profundidade da mossa na probabilidade $\begin{array}{ll}\text { de falha do dano mossa-sulco } & 115\end{array}$

5.2.4. Influência da profundidade do sulco na probabilidade de falha do dano mossa-sulco 
6. Conclusões e considerações finais

7. Referências bibliográficas

121

Apêndice A

Aplicação da análise de valor extremo em dutos com

defeitos de corrosão

131 


\section{Lista de figuras}

Figura 2.1. Fundamento da integridade estrutural 28

Figura 2.2. Distribuição normal da função de estado limite $m$

Figura 2.3. Representação da superfície de falha no espaço padronizado

Figura 2.4. Método FORM algoritmo HR-LF

Figura 2.5. (a) Sistema em série. (b) Sistema em paralelo

Figura 3.1. Função Binormal

Figura 3.2. Influência do coeficiente de correlação na função de densidade de probabilidade padrão binormal

Figura 3.3. Cálculo da POF para um sistema com duas Funções limites. Usando a integral da função de densidade de probabilidade padrão binormal

Figura 3.4. Cálculo da POF para um sistema em série

Bidimensional (a) Região de falha verdadeira eq. (3.41)

(b) Espelho da região de falha usado pelo PCM eq. (3.40)

Figura 4.1. Distribuição das medições de um PIG com Exatidão de $\pm 10 \%$ de $t$, com nível de confiança de $80 \%$

Figura 4.2. Curva de $P O D$ para um PIG Magnético (MFL)

Figura 4.3. Curva de $P O D$ para técnica de inspeção

por partículas magnéticas $(M P l)$ em baixo d'água

Figura 4.4. Estados limites para dutos com defeitos de corrosão

Figura 4.5. Geometria de um defeito de corrosão

Figura 4.6. Planilha EXCEL para o cálculo da probabilidade de falha

Figura 4.7. Fator de importância de cada variável na $P O F$ por ruptura

Figura 4.8. Fator de importância de cada variável na POF por vazamento 
Figura 4.9. Modelos mais comuns que descrevem a perda $\begin{array}{ll}\text { por corrosão } & 77\end{array}$

Figura 4.10. Modelo de perda por corrosão

Figura 4.11. Cálculo da POF seguindo um modelo de corrosão com atualização (inspeção) e sem atualização

Figura 4.12. Influência do modelo de corrosão no cálculo da POF

Figura 4.13. Crescimento da trinca na chapa 91

Figura 4.12. Atualização da POF 93

Figura 5.1. Dano mecânico Mossa-Sulco 96

Figura 5.2. Diagrama de Falha (FAD - Failure Assessment

Diagram)

Figura 5.3. Planilha EXCEL para o cálculo da POF devido ao dano mossa-sulco

Figura 5.4. Diagrama de falha para o dano mossa-sulco com o ponto mais provável de falha

Figura 5.5. Fator de importância de cada variável na $P O F$ por ruptura

Figura 5.6. Freqüência de falha em função do diâmetro do duto 105

Figura 5.7. Influência de $L_{s}$ na $P O F$ do dano mossa-sulco

Figura 5.8. Influência de $L_{s}$ na avaliação do dano mossa-sulco usando o $F A D$

Figura 5.9. Região de vazamento e de ruptura no $F A D$

Figura 5.10. Planilha EXCEL para o cálculo da probabilidade de $L_{c}-L_{s} \leq 0$ e $L_{c}-L_{s}>0$

Figura 5.11. Fator de importância de cada variável na POF por ruptura

Figura 5.12. Influência da pressão de operação da $P O F$ de um duto com defeito mossa-sulco

Figura 5.13. Influência da pressão de operação no $F A D$ de um duto com defeito mossa-sulco

Figura 5.14. Influência da profundidade da mossa na $P O F$ 
de um duto com defeito mossa-sulco

Figura 5.15. Influência da profundidade da mossa no $F A D$

de um duto com defeito mossa-sulco

Figura 5.16. Influência da profundidade do sulco na $P O F$

de um duto com defeito mossa-sulco

Figura 5.17. Influência da profundidade da mossa no $F A D$

de um duto com defeito mossa-sulco

Figura 6.1. Programa para o gerenciamento da confiabilidade estrutural de dutos corroídos 


\section{Lista de tabelas}

Tabela 4.1 Variáveis do duto AA API 5L X52 73

$\begin{array}{ll}\text { Tabela 4.2 Dados do defeito } & 73\end{array}$

Tabela 4.3 Dados da inspeção (PIG MFL 80\% de confiança) 73

$\begin{array}{ll}\text { Tabela 4.4 Resultados das análises de POF } & 74\end{array}$

Tabela 4.5 Resultados das análises de POF com vários

$\begin{array}{ll}\text { defeitos } & 76\end{array}$

Tabela 4.6 Resultados das análises de POF 83

Tabela 4.7 - POF admissível ULS segundo a DNV RP F-101 84

Tabela 4.8 - $\operatorname{POF}\left(g_{I} \geq 0\right)$ para o ano $14 \quad 85$

Tabela 4.9 - Variáveis $\quad 88$

Tabela 4.10 Resultados das análises de POF 88

Tabela 4.11 - $\operatorname{POF}\left(g_{I} \geq 0\right)$ para o ano $7 \quad 89$

Tabela 5.1 Variáveis usadas no cálculo da POF 102

Tabela 5.2 Freqüência de Falha usando confiabilidade $\begin{array}{ll}\text { estrutural } & 104\end{array}$

Tabela 5.3 Freqüência de Falha de bases de dados 105

Tabela 5.4 Cálculo da probabilidade de $L_{c}-L_{s} \leq 0$ e $L_{c}-L_{s}>0 \quad 112$

Tabela 5.5 Cálculo da POF por ruptura e vazamento 112

Tabela 5.6 Cálculo da POF total 112 